\title{
Development of modern CANDU PHWR cross-section libraries for SCALE
}

\author{
Nathan T. Shoman ${ }^{\mathrm{a}, *}$, Steven E. Skutnik ${ }^{\mathrm{a}, * *}$ \\ ${ }^{a}$ Department of Nuclear Engineering, University of Tennessee-Knoxville, Knoxville, TN, 37996
}

\begin{abstract}
A new set of SCALE fuel lattice models have been developed for the 28-element and 37-element CANDU fuel assembly designs using modern cross section data from ENDF-B/VII.0 in order to produce new reactor data libraries for SCALE/ORIGEN depletion analyses. These new libraries are intended to provide users with a convenient means of evaluating depletion of CANDU fuel assemblies using ORIGEN through pre-generated cross sections based on SCALE lattice physics calculations. The performance of the new CANDU ORIGEN libraries in depletion analysis benchmarks to radiochemical assay data were compared to the previous version of the CANDU libraries provided with SCALE (based on WIMS-AECL models).

Benchmark comparisons with available radiochemical assay data indicate that the new cross-section libraries perform well at matching major actinide species $(\mathrm{U} / \mathrm{Pu})$, which are generally within $1-4 \%$ of experimental values. The library also showed similar or better results over the WIMS-AECL library regarding fission product species and minor actinoids ( $\mathrm{Np}, \mathrm{Am}$, and $\mathrm{Cm}$ ). However, a notable exception was in calculated inventories of ${ }^{154} \mathrm{Eu}$ and ${ }^{155} \mathrm{Eu}$, where the new library employing modern nuclear data (ENDF/B-VII.0) performed substantially poorer than the previous WIMS-AECL library (which used ENDF-B/VI.8 crosssections for these species). The cause for this discrepancy appears to be due to differences in the ${ }^{154} \mathrm{Eu}$ thermal capture cross-section between ENDF/B-VI.8 and ENDF/B-VII.0, an effect which is exacerbated by the highly thermalized flux of a CANDU heavy water reactor compared to that of a typical light-water reactor spectrum.

Overall, the new SCALE-based CANDU libraries appear to give very good agreement to experimental benchmarks, indicating that the new libraries should provide satisfactory depletion calculation performance generally comparable or better to the prior WIMS-AECL libraries.
\end{abstract}

Keywords: CANDU, PHWR, SCALE, ORIGEN, Reactor Physics, Nuclear Fuel Depletion

\section{Introduction}

The purpose of this project was to develop a working set of CANada Deuterium Uranium (CANDU) pressurized heavy water reactor (PHWR) fuel lattice models for cross section library production based on modern nuclear data (ENDF/B-VII.0) compatible with the latest SCALE physics modules [1, 2]. Cross section libraries for the CANDU 28-element and 37-element designs were produced for use in the ORIGEN depletion sequence [3].

\footnotetext{
${ }^{*}$ Corresponding author

${ }^{* *}$ Principal corresponding author

Email addresses: nshoman@vols.utk.edu (Nathan T. Shoman), sskutnik@utk.edu (Steven E. Skutnik)
} 
In this paper, the means of developing these CANDU lattices for reactor data libraries are presented, including details of the lattice construction, validation studies to characterize the performance of the new libraries compared to both radiochemical assay data as well as the prior version of the library, and refinements made to the model to ensure maximum fidelity of the new lattice model. The computed isotopic inventories using these new models were then checked against previously developed benchmarks based on radiochemical assay data and found to show good agreement.

\section{Motivation}

SCALE includes a number of pre-generated reactor-specific data libraries based upon lattice physics calculations for use with the ORIGEN depletion module [3]. These libraries are intended to allow users to perform rapid depletion calculations without the need to re-run computationally expensive lattice physics transport calculations, namely by providing a set of pre-calculated one-group cross-sections for specific initial assembly configurations at set burnup intervals. These pre-calculated libraries can then be interpolated to problem-specific conditions (e.g., initial enrichment, cycle burnup, etc.) for use with ORIGEN [2, 3]

The reactor data libraries provided with SCALE include most major U.S. light water reactor (LWR) fuel assembly types, as well as MOX-based pressurized water reactor (PWR) and boiling water reactor (BWR) fuel assembly configurations along with several foreign reactor types, such as RBMK, VVER, Magnox, and CANDU PHWR lattice types. These libraries allow for rapid evaluation of fuel depletion problems by interpolating cross-sections calculated from prior 2-D transport calculations to problem-specific conditions (e.g., enrichment, moderator density, and burnup), thus largely preserving solution accuracy.

While current and previous versions of SCALE provide CANDU 28-element and 37-element crosssection libraries for use with ORIGEN, no current lattice templates compatible with the NEWT geometry processor currently exist. Meanwhile, the previous libraries contain a very limited burnup region, ranging from $230 \mathrm{MWd} / \mathrm{MTU}$ to $12,480 \mathrm{MWd} / \mathrm{MTU}$ [3, 4]. Because no initial zero-burnup cross-sections are provided on the included CANDU libraries, ORIGEN cannot interpolate any data in the 0 to $230 \mathrm{MWd} / \mathrm{MTU}$ region with the provided libraries.

Additionally, the previous libraries were generated using the two-dimensional transport code WIMSAECL using the ENDF/B-V nuclear data [5, 6]. The new libraries have been evaluated using modern nuclear data (ENDF/B-VII.0) and the current SCALE 2-D deterministic transport sequence, NEWT [7]. As part of this work, templates have been developed that are compatible for both a Monte-Carlo approach via the KENO sequence [8] or a discrete-ordinates sequence via NEWT.

\section{Experimental Assay Data}

Validation of the new CANDU reactor data libraries for ORIGEN was conducted by comparing calculated inventories from depletion to known radiochemical assay benchmark studies for CANDU fuel assemblies. Measurements were available for both Pickering-A and Bruce-A fuel assembly nuclide inventories. Available radiochemical assay data includes studies on CANDU assemblies from two reactors: Bruce-A, a 37-element assembly benchmark which was limited to uranium and plutonium isotopic ratios, and PickeringA, which included several important fission products and minor actinoids [6, 5].

The Bruce-A assay provides measurements from each fuel ring in the assembly which can be used to construct assembly average isotopic values. However, the Pickering-A assay only includes isotopic measurements from the outer fuel ring. These results are not directly applicable to benchmarking the homogenized one group cross-sections. A number of different benchmarks are provided in an attempt to provide an adequate comparison. 


\subsection{Bruce-A Fuel Nuclide Inventory}

Isotopic measurements on fuel bundle F21037C from the Bruce-A Nuclear Generating Station were obtained as part of the Thorium Fuel Reprocessing Experiment Program conducted by the AECL in 1982 [5]. The 37-element CANDU fuel design consists of a single central pin surrounded by three concentric rings of fuel pins, each with 6,12 , and 18 pins, respectively. The assay was taken from multiple pins from different rings within the bundle. These measurements were combined to determine assembly-averaged inventories. The central fuel pin was not assayed, however, it was assumed to have the same fuel composition as the inner ring [6, 5]. The discharge burnup of the bundle was approximately $7980 \mathrm{MWd} / \mathrm{kgU}$.

\subsection{Pickering-A Fuel Nuclide Inventory}

For the 28-element assembly benchmark, a single fuel assay was performed on an outer element of Pickering-A fuel bundle 19558C [5]. The measurements were made to provide a benchmark base for codes used to predict used fuel inventories for the Waste Management Program at AECL. The bundle consisted of 28 natural uranium oxide pellets, each surrounded by Zircaloy-4 cladding. The bundle had a discharge burnup of $9208 \mathrm{MWd} / \mathrm{kgU}$.

The original benchmarking study reported that the relatively large discrepancies in ${ }^{99} \mathrm{Tc}$ and ${ }^{106} \mathrm{Ru}$ could be attributed to significant quantities remaining in undissolved residue after recovery, indicating this could likely be due to poor counting geometries used in analyzing the residues [6]. The report further indicated that errors in ${ }^{129}$ I could similarly be attributed to measurement issues resulting from the difficulty in capturing off-gasses during the sample dissolution.

The WIMS-AECL lattice evaluations employed ENDF/B-V for fission yields whereas nuclear decay and energy release data was based on ENDF/B-VI. The original WIMS-AECL library was a hybrid containing ENDF/B-V cross sections, supplemented with ENDF/B-VI cross sections for a number of nuclides not available in ENDF/B-V. Additionally, ENDF/B-VI cross sections were used for ${ }^{154} \mathrm{Eu}$ and ${ }^{155} \mathrm{Eu}$ due to large differences in the resonance energy region [6].

The ratio of the WIMS-AECL cluster-averaged inventories to outer element inventories was used to scale the measured data in order to obtain an estimate of the cluster-averaged inventory. The scaled fission products were not reproducible using this method, so the WIMS-AECL scaled values were used instead of TRITON scaled values from the new lattice. The ratio by which the inventories are scaled was not provided in the primary report. Caution must be used as using this method introduces a bias. If the ratios of the outer inventory to the cluster-averaged values are incorrect for any reason it will impact the comparison. This comparison is nonetheless considered due to a lack of available cluster-averaged data. It should be noted that not all of the fission products were scaled. Nuclides with a small capture cross section and for which the dominant production route is fission yield will not be significantly impacted by error induced by assembly-averaged cross-sections and flux.

\subsubsection{Discrepancies in Pu-241 inventories due to errors in reported decay times}

Originally, the ${ }^{241} \mathrm{Pu}$ inventories for both assemblies were incorrect. The $37-e l e m e n t$ bundle ${ }^{241} \mathrm{Pu}$ inventory was under-predicted by $35-40 \%$ while the 28 -element ${ }^{241} \mathrm{Pu}$ inventory was over-predicted by approximately $70 \%$. It was eventually determined that there was an error in the radiochemical benchmark reference paper [6] used to evaluate these models. The original benchmark report [6] reported a final decay time after discharge of 5590 days for the the 37-element Bruce-A assembly (Table 20 in the original report), while the 28-element Pickering-A assembly had a decay time prior to measurement of 1162 days (Table 23 of the original report). It was not possible to reproduce their results with this data. However, a separate study referencing the radiochemical assay performed on the Pickering-A (28-element) fuel assembly [4, 5] contradicts this decay time, indicating a final decay step of 5590 days prior to measurement (rather than 1162 days), 
implying that the decay times for the two assemblies had inadvertently been reversed. When these changes are made in the TRITON model, the models showed substantially improved agreement with the isotopic benchmark, particularly for ${ }^{241} \mathrm{Pu}$. The correct decay time was confirmed via private correspondence with the author of the original benchmarking study.

The corrected power histories for each assembly are presented in Tables 1 and 2 . Two power histories are provided for the 28 -element lattice, one for the assembly power history and an inferred power history reported for the outer element [6]. This outer element power history was used with the WIMS-AECL transport sequence to control the specific burnup of the outer element for the 28-element bundle, due to the fact that the radiochemical assay benchmark was based upon measurements of a single outer-element pin. This history is thus provided here in addition to the nominal assembly power history for benchmarking purposes.

Table 1: Power history used for modeling the Pickering-A CANDU 28-element bundle (with corrected final decay time) for the nominal assembly power and the calculated outer element power [5, 6].

\begin{tabular}{c|ccc}
\hline $\begin{array}{c}\text { Irradiation } \\
(\text { days })\end{array}$ & \multicolumn{2}{|c}{ Power $\left(\frac{\mathrm{MW}}{\mathrm{MTU}}\right)$} & Cooling \\
\hline 31.33 & 28.41 & 31.4 & 3.25 \\
15.38 & 26.26 & 28.9 & 5.61 \\
66.67 & 28.15 & 31.1 & 0.00 \\
66.67 & 26.71 & 29.5 & 9.00 \\
64.55 & 26.07 & 28.7 & 15.0 \\
70.78 & 24.18 & 26.7 & 5590.0 \\
\hline
\end{tabular}

Table 2: Power history used for modeling the Bruce-A CANDU 37-element bundle (with corrected final decay time) [5, 6].

\begin{tabular}{ccc}
\hline $\begin{array}{c}\text { Irradiation } \\
\text { (days) }\end{array}$ & $\begin{array}{c}\text { Power } \\
\left(\frac{\mathrm{MW}}{\mathrm{MTU}}\right)\end{array}$ & $\begin{array}{c}\text { Cooling } \\
\text { (days) }\end{array}$ \\
\hline 31.63 & 1.040 & 0.0 \\
13.00 & 30.10 & 10.0 \\
43.25 & 30.21 & 55.3 \\
49.75 & 30.30 & 9.0 \\
50.33 & 30.30 & 15.0 \\
103.00 & 30.30 & 3.0 \\
117.25 & 0.870 & 1162.0 \\
\hline
\end{tabular}

\section{Lattice Development}

Because NEWT does not support triangular-pitch repeated-element arrays, modeling the CANDU assemblies as a true repeated-element lattice proved impossible. Alternative geometries (such as hexagonalpitch arrays) are unable to correctly represent the non-uniform radial spacing between circular arrays of elements; i.e., the distance from the inner ring to the center ring is not the same as that from the middle ring to the outer ring. 
Table 3: Geometry specification for CANDU 28-element bundle lattices. Phase denotes the angular position of the first element in the assembly, wherein each subsequent element is offset by angle $\Delta \theta$.

\begin{tabular}{rrrr} 
Ring & $\mathrm{r}(\mathrm{cm})$ & $\Delta \theta\left(^{\circ}\right)$ & Phase $\left(^{\circ}\right)$ \\
\hline Inner & 1.175 & 90 & 45.0 \\
Middle & 2.685 & 45 & 22.5 \\
Outer & 4.229 & 22.5 & 11.25 \\
\hline
\end{tabular}

Table 4: Geometry specification for CANDU 37-element bundle lattices. Phase denotes the angular position of the first element in the assembly, wherein each subsequent element is offset by angle $\Delta \theta$.

\begin{tabular}{rrrr}
\hline Ring & $\mathrm{r}(\mathrm{cm})$ & $\Delta \theta\left(^{\circ}\right)$ & Phase $\left(^{\circ}\right)$ \\
\hline Center & 0 & - & - \\
Inner & 1.4885 & 60 & 0.0 \\
Middle & 2.8755 & 30 & 15.0 \\
Outer & 4.3305 & 20 & 0.0 \\
\hline
\end{tabular}

The only available modeling solution was to insert each individual fuel element into the model as holes. Coordinates for each hole were determined by establishing the ring radius and the angular spacing $(\Delta \theta)$ between each element (assuming an equal angular spacing of elements for each ring), along with an appropriate angular offset between rings (i.e., a "phase" angle). Determining the correct "phase angle" between elements of individual rings is necessary to ensure the correct line-of-sight of fuel elements between rings, which will influence the result (e.g., as indicated by the Dancoff Factors, discussed in the next section). The relationships for the ring positions, such as the ring radius, angular offset between elements $(\Delta \theta)$, and the "phase" of each ring (defined as the angular position of the first element in the ring) are presented for the 28 and 37-element assemblies in Tables 3 and 4 , respectively.

The final 28-element and 37-element lattice models are shown as Figures 1(a) and Figure 1(b) To maximize the accuracy of the multi-group transport (NEWT) solution, a tighter mesh was imposed inside the pressure tube and calandria along with within the fuel elements themselves. Further, to ensure a correct representation of each assembly's relative orientation, the final models (Figures 1(a) and (Figure 1(b) were likewise compared to available images of each assembly's orientation [5, 6, 9].

\subsection{Dancoff Factors}

With the correct geometry model for the fuel assemblies established, it is important to determine the Dancoff factors for multi-group deterministic transport calculations (like NEWT). The Dancoff factor is the probability that a neutron emitted isotropically from the surface of one absorber lump will pass through the external media and enter a nearby absorber lump [10]. (Here it should be noted that Dancoff Factors apply exclusively to multi-group transport calculations; such a correction is not required for continuous-energy Monte Carlo approaches like KENO). Dancoff factors have a significant impact on transport calculation (including for isotopic calculations), given the relatively heterogeneous environment of the CANDU fuel assembly. This finding is consistent with prior studies evaluating impacts on other heterogeneous assembly types, such as SVEA BWR assembly types [11]. The study found that differences in Dancoff factors can have large impacts in the effective cross sections, and thus, the isotopic calculations. It was also noted that at higher burnups Dancoff factors are particularly important. Deviations of $8 \%$ for ${ }^{235} \mathrm{U}$ and 3-5\% in plutonium isotopics were seen when Dancoff factors were not used. 


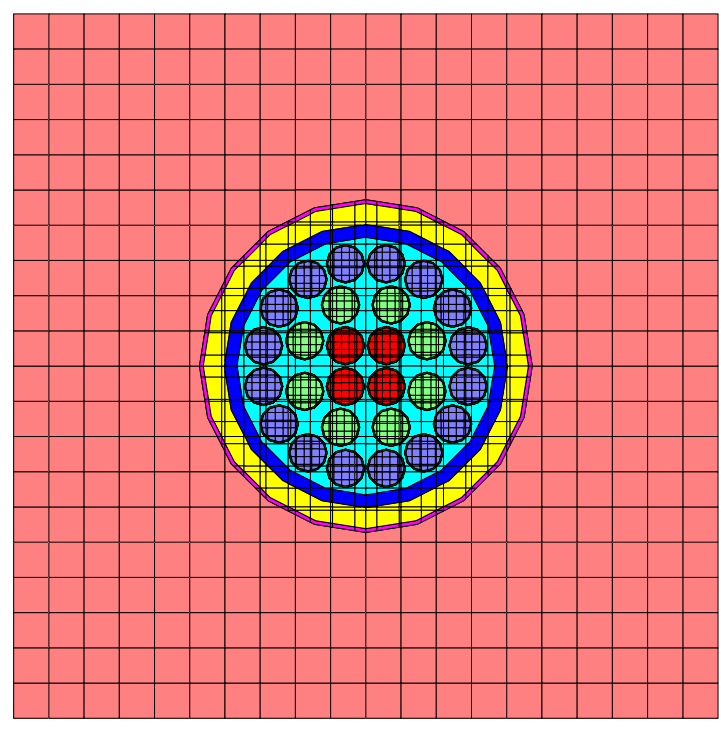

(a) CANDU 28-element bundle

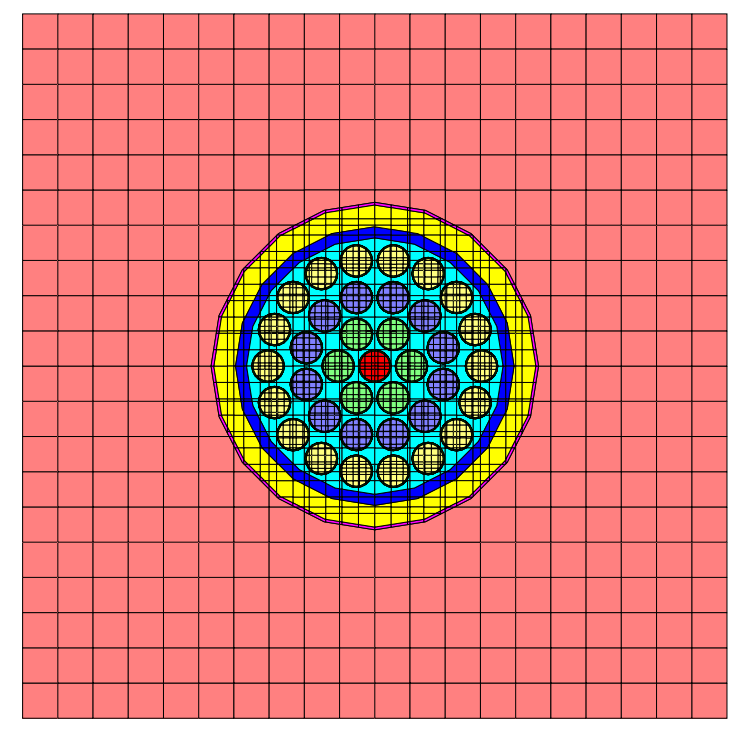

(b) CANDU 37-element bundle

Figure 1: Final holes-based geometries for CANDU assembly lattices

Table 5: Calculated Dancoff Factors for CANDU 28-element and 37-element assemblies

\begin{tabular}{lll} 
& \multicolumn{2}{c}{ Dancoff Factor } \\
Position / Ring & 28-element & 37-element \\
\hline Center pin & - & 0.763 \\
Inner ring & 0.714 & 0.761 \\
Middle ring & 0.707 & 0.728 \\
Outer ring & 0.447 & 0.442 \\
\hline
\end{tabular}

Dancoff factors were analyzed to using the MCDancoff module to determine a ring-averaged Dancoff factor. Included in Table 5 are the calculated ring-averaged Dancoff Factors for both the 28-element and 37-element CANDU assembly types. From Table 5, one apparent feature of both lattices is the fact that the outer-ring Dancoff factor is substantially lower than those of the inner bundle rings (indicating a relatively higher probability of absorption). Here, this can be understood with respect to the average moderation of neutrons in the outer-ring elements compared to the inner rings. Neutrons created in other bundles will be highly thermalized; likewise, given the solid angle subtended by the outer ring elements, they have a comparatively high rate of absorption in the outer elements. This higher rate of absorption will thus manifest as a comparatively higher power in the outer ring elements compared to the bundle average.

\subsection{Model parameters}

The modeling parameters used for transport and depletion calculations are presented in Table 6 . In order to achieve maximum accuracy in isotopic predictions, four depletion subintervals were used per depletion cycle (nlib=4). Trace levels of 230 nuclides were added to the fuel mixtures (addnux=3) in order to 
accurately account for the effect of key isotopes on depletion calculations (especially at higher burnups). Parameters specific to multi-group transport included the convergence criteria $(\epsilon), \mathrm{P}_{\mathrm{N}}$ scattering order, and coarse-mesh finite differencing (CMFD, used to accelerate source convergence).

Table 6: Modeling parameters used for both CANDU assembly lattices

\begin{tabular}{|c|c|c|}
\hline Parameter & Value & Description \\
\hline addnux & 3 & $\begin{array}{l}\text { Add trace levels of } 230 \text { nuclides to the fuel mixture composition at the start of } \\
\text { depletion (to ensure inclusion in the first cross-section update) }\end{array}$ \\
\hline$\epsilon$ & $8.0 \mathrm{E}-4$ & Source convergence criteria for multi-group deterministic transport \\
\hline CMFD & yes & Coarse-mesh finite differencing acceleration \\
\hline$x y c m f d$ & $4 \times 4$ & Four fine mesh cells per coarse mesh cell (for CMFD acceleration) \\
\hline $\mathrm{P}_{\mathrm{N}}$ & $2 / 1$ & $\mathrm{P}_{\mathrm{N}}$ scattering order; set to 2 for moderator and coolant, 1 for all other materials \\
\hline nlib & 4 & Number of depletion sub-intervals per irradiation cycle \\
\hline
\end{tabular}

In order to accurately validate the model, results from both deterministic and Monte Carlo methods were compared to known assay data. Monte Carlo results were obtained by using the KENO transport model and the discrete ordinates results were obtained using TRITON/NEWT. Additionally, the effect of employing ENDF/B-V versus ENDF/B-VII.0 cross-section data for the models was compared. In summary, a total of four data sets were generated for each assembly.

\section{Lattice physics validation studies}

The primary method of validation for the model was to compare the computational results from the developed model to measured isotopic data from radiochemical assay experiments. Two such studies were available [4, 6], and as such, these studies were used to benchmark the performance of the new fuel bundle lattice models. Further, the new lattice models were similarly benchmarked against the performance of the prior WIMS-AECL model (which served as the basis for the prior CANDU-based cross-section libraries distributed with SCALE) by comparing their isotopic calculations the WIMS model [5]. The WIMS-AECL lattice was unavailable for use so reported values were used in lieu of calculated values. The computational results are presented as $(\mathrm{C} / \mathrm{E})-1$ values. Error bars are provided to represent the one-sigma experimental uncertainty in radiochemical assay values.

\subsection{8-element bundle benchmark}

A single benchmark based on transport calculations are conducted for the 28 -element lattice. The aim of the transport benchmark is to test the accuracy of the lattice parameters such as geometry, materials, and Dancoff factors. The library benchmarks objective is to determine the performance of the ORIGEN library produced from the 28-element lattice.

For the transport benchmark, the outer assembly element isotopics were calculated through TRITON/NEWT and TRITON/KENO by depleting each ring of fuel-elements individually, which was necessary in the multigroup transport solution due to ring-specific Dancoff factors. NEWT and KENO both provide ring-specific isotopic measurements from which the outer ring was compared. This benchmark will indicate how well the lattice geometry performs and determine how well the transport method distributes the power among the rings. For the first benchmark (referred to hereout as the "nominal" benchmark), the nominal assembly power history in Table 1 was used for the total assembly power, where power in the individual depletion materials was allowed to float (conserving total assembly power). Here, the isotopic benchmark was calculated 
by comparing the outer ring inventory to the measured outer ring inventory from the radiochemical assay studies.

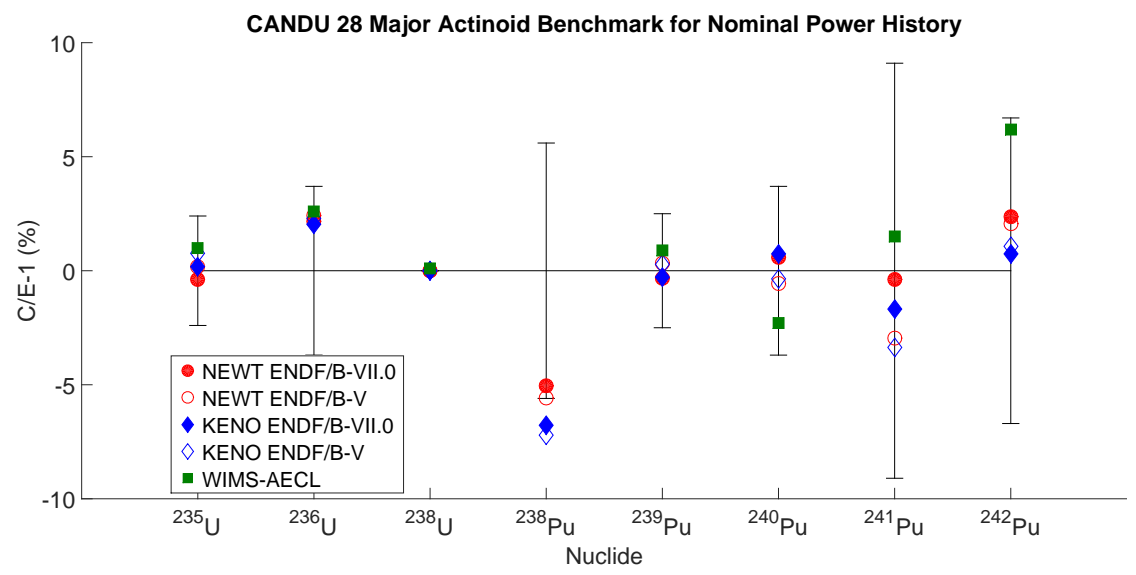

Figure 2: CANDU 28-element outer-ring depletion benchmark (based on Pickering-A outer-ring data using the nominal assembly power history) for major actinoids [5] 6

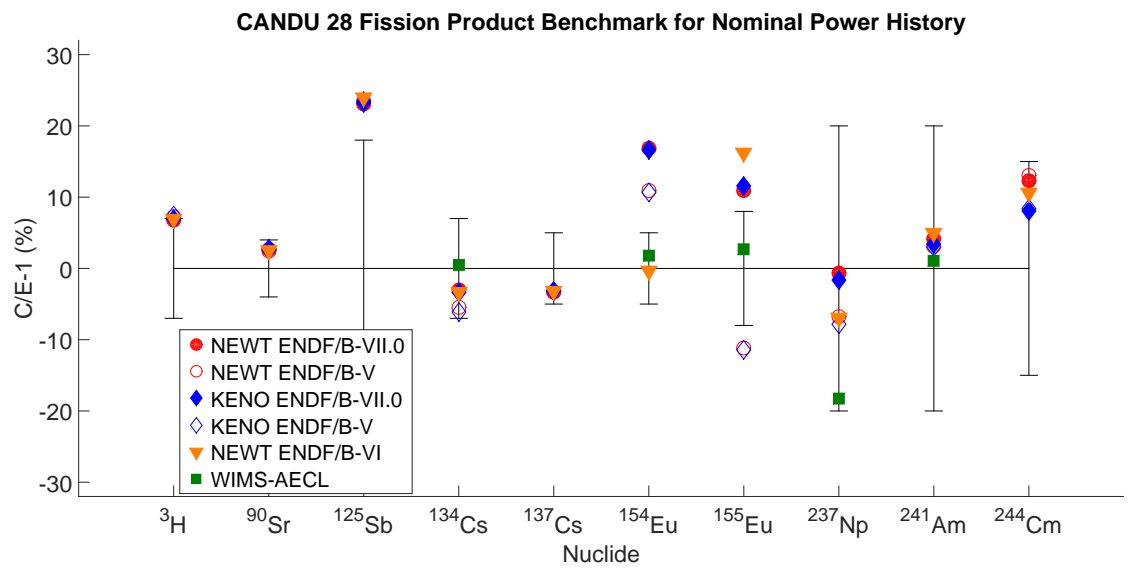

Figure 3: CANDU 28-element outer-ring depletion benchmark (based on Pickering-A outer-ring data using the nominal assembly power history) for fission products and minor actinoids [5] 6

\subsubsection{Nominal assembly power benchmark}

For the nominal assembly power benchmark, the assembly was depleted using the average assembly power, where powers of individual assembly elements were allowed to float, preserving total assembly power (i.e., the assembly power was normalized to the nominal power history expressed in Table 1). Validation was performed by comparing the calculated outer ring isotopic inventory to the measured outer ring inventory. The benchmark was run with Monte Carlo (KENO) and Discrete Ordinate (NEWT) transport using ENDF/B-V and ENDF/B-VII.0 cross-sections. An ENDF/B-VI.8 benchmark was also conducted with the 
new lattice to evaluate the effect of cross-section libraries on the production of the europium isotopes ${ }^{154} \mathrm{Eu}$ and ${ }^{155} \mathrm{Eu}$, but only using the NEWT transport sequence.

Both ENDF/B-V.2 and ENDF/B-VII.0 produced similar results for the major actinides (Figure ??). However, ENDF/B-VII.0 showed slightly improved agreement to the benchmark values, which is consistent with prior comparisons for PWR assemblies [12]. Although many of the minor actinoids and fission products showed comparatively high error, several of the errors can be explained by issues with the experimental procedure described in Section 3.2 However, there remains good agreement with key burnup indicators (Figure ??), such as ${ }^{137} \mathrm{Cs}$ and ${ }^{134} \mathrm{Cs}$, thus indicating that the transport-calculated flux is likely accurate. It is important to note that unlike both ENDF/B-V.2 and ENDF/B-VII.0, ENDF/B-VI.8 did produce accurate values for ${ }^{154} \mathrm{Eu}$.

Many of the minor actinoids and fission products, such as ${ }^{244} \mathrm{Cm}$, were not available for comparison with the WIMS-AECL code, due to missing nuclides in the WIMS-AECL library. With respect to the measured isotopes, the new 28 -element lattice appears to produce accurate inventories for the major actinides regardless of the cross-section library used. There was a significant improvement in ${ }^{237} \mathrm{~Np}$ accuracy over WIMS-AECL in the new lattice, as well as ${ }^{238} \mathrm{Pu}$.

Here, accurate assessment of many of the transuranic species is especially important for used nuclear fuel disposition studies. ${ }^{237} \mathrm{~Np}$ is a nuclide of particular concern from a modeling standpoint for geologic disposal evaluations; under certain geochemical conditions, ${ }^{237} \mathrm{~Np}$ can act as a significant dose contributor, given its relatively high solubility and mobility in groundwater, while ${ }^{244} \mathrm{Cm}$ acts as a significant neutron source for used fuel storage.

\subsection{7-element bundle benchmark}

Here, the most accurate experimental data were for the uranium and plutonium atom ratios. Like the Pickering-A bundle, the uranium and plutonium ratios were selected for comparison. The (C/E)-1 values are likewise presented in Figure 4 . However, unlike the 28-element bundle, a detailed assay of the fission products was not performed on the 37-element bundle, thus an extended isotopic comparison cannot be made.

\subsubsection{Nominal assembly power benchmark}

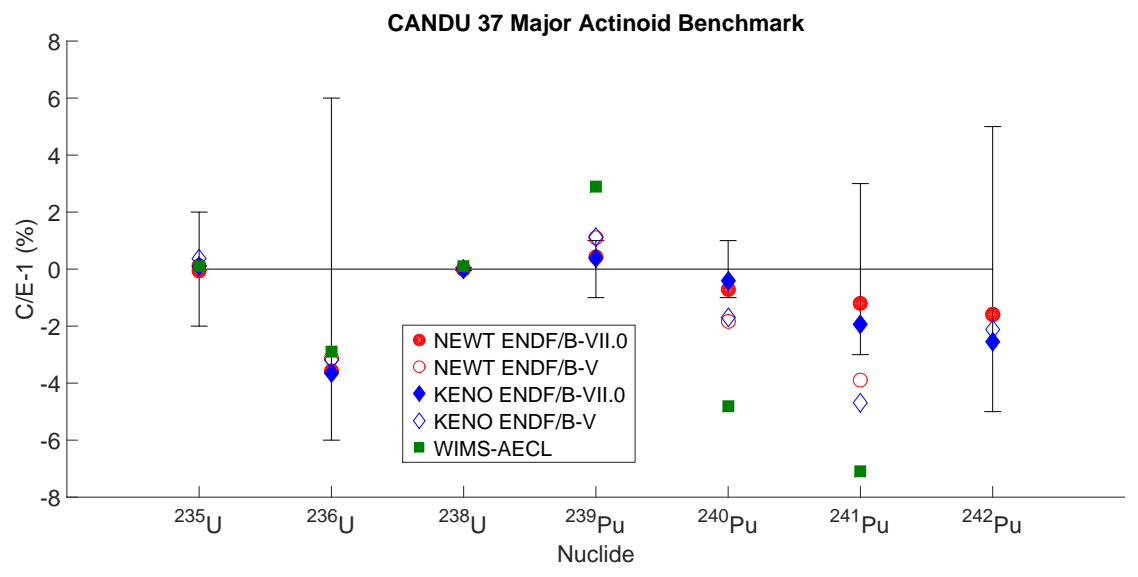

Figure 4: CANDU 37-element bundle depletion benchmark (based on Bruce-A assembly data) for major actinoids [5] 6]. 
NEWT and KENO produced very similar results for both ENDF/B-VII.0 and ENDF/B-V.2 with the 37element lattice. The ENDF/B-VII.0 library produced more accurate results for many of the major actinoids, with the exception of ${ }^{236} \mathrm{U}$. The largest discrepancy between the libraries is less than $3 \%$, which is consistent with prior literature [12]. It should be noted that the 37-element isotopic assay was determined from several samples from each ring and combined into an assembly-average value which enabled an assembly wide comparison.

The most accurate 37-element lattice model involved using the NEWT transport sequence and ENDF/BVII.0 library. The resulting calculated inventory lies within the experimental uncertainty, with the results for the ENDF/B-V model residing just outside the experimental uncertainty for the plutonium isotopes. The new lattice produced accurate values that were inside experimental uncertainty for all assayed major actinides. This is an improvement over the WIMS-AECL based lattice where ${ }^{241} \mathrm{Pu}$ and ${ }^{242} \mathrm{Pu}$ were calculated outside the experimental uncertainty.

\subsection{Europium inventory production}

The previous WIMS-based 28-element library was able to achieve reasonable prediction of ${ }^{154} \mathrm{Eu}$ and ${ }^{155}$ Eu by using ENDF/B-VI cross-sections for these isotopes. The ENDF/B-VI.8 library did produce accurate inventory predictions for ${ }^{154} \mathrm{Eu}$ (with a difference of about $1.34 \%$, well within experimental uncertainty), however ${ }^{155} \mathrm{Eu}$ was still predicted higher than the experimental value by about $15 \%$. One plausible explanation for this deviation is due to the evaluated radiative capture cross-sections for Eu species in the thermal region, and in particular the differences in evaluated capture cross-section values between ENDF/B-V.2, VI.8, and VII.0. The ENDF/B-VI.8 cross-sections for ${ }^{154} \mathrm{Eu}$ produced good agreement for ${ }^{154} \mathrm{Eu}$, however, ${ }^{155} \mathrm{Eu}$ was not produced within the measurement uncertainty bounds for any cross-section library.

To understand this issue, it is first important to understand the production and loss channels for Eu species, which can be represented by the general expression in Equation 1

$$
\frac{d N_{i}}{d t}=\overbrace{\sum_{m=1}^{M} Y_{m} \lambda_{m} N_{m}}^{\text {A }}+\underbrace{\phi \sigma_{i-1}^{a} N_{i-1}}_{\text {B }}-\overbrace{\lambda_{i} N_{i}}^{\mathrm{C}}-\underbrace{\phi \sigma_{i}^{a} N_{i}}_{\text {(D) }}+\overbrace{Y_{i} \Sigma_{f} \phi}^{\mathrm{E}}+\underbrace{\sum_{m \neq i} N_{m} \sigma_{m \rightarrow i} \phi}_{\text {F }}
$$

where

- Term $\mathrm{A}=$ Production of nuclide $i$ from $M$ parent isotope decays

- Term $\mathrm{B}=$ Production of nuclide $i$ from neutron absorption

- Term $\mathrm{C}=$ Loss of nuclide $i$ to decay

- Term $\mathrm{D}=$ Loss of nuclide $i$ to neutron absorption

- Term $\mathrm{E}=$ Direct production of nuclide $i$ from fission

- Term $F=$ Other neutron-induced reactions (e.g., $(n, p),(n, 2 n)$, etc.)

The dominant terms controlling the ${ }^{154} \mathrm{Eu}$ and ${ }^{155} \mathrm{Eu}$ inventories are A, B , and (D). Given that the direct fission yields of ${ }^{154} \mathrm{Eu}$ and ${ }^{155} \mathrm{Eu}$ from ${ }^{235} \mathrm{U}$ and ${ }^{239} \mathrm{Pu}$ are both vanishingly small, the predominant production channels for both species are therefore through parent isotope decays (e.g., for ${ }^{155} \mathrm{Eu}$ from the decay 
of ${ }^{155} \mathrm{Sm}$ ) as well as through successive neutron capture reactions, i.e. ${ }^{153} \mathrm{Eu}(\mathrm{n}, \gamma){ }^{154} \mathrm{Eu}$ and ${ }^{154} \mathrm{Eu}(\mathrm{n}, \gamma){ }^{155} \mathrm{Eu}$. ${ }^{153} \mathrm{Eu},{ }^{154} \mathrm{Eu}$, and ${ }^{155} \mathrm{Eu}$ all have relatively high thermal-region neutron capture cross-sections, ranging from about 335 barns for ${ }^{153} \mathrm{Eu}$ [13] to several thousand barns for ${ }^{154} \mathrm{Eu}$ and ${ }^{155} \mathrm{Eu}$ (with even higher capture cross-sections in the resonance energy regions), making capture a predominant production and loss channel for these species.

To understand the relative contributions to nuclide production and loss, it is helpful to refer to the ORIGEN transition matrix $\overline{\bar{A}}$, which describes the total transition rate constant for each set of coupled differential equations describing the system, i.e. [3]:

$$
\frac{d \bar{N}}{d t}=\overline{\bar{A}} \cdot \bar{N}(t)
$$

The transition matrix $\overline{\bar{A}}$ describes the transition rate coefficients for each isotopic transition from neutron reactions and decay. Thus, each individual element of $\overline{\bar{A}}$ (denoted $a_{i j}$ ) describes the relative transition rate from nuclide $i$ to $j[3]$ :

$$
a_{i j}=-\ell_{i j} \lambda_{i}-f_{i j} \bar{\phi} \sigma_{i}
$$

Where $\ell_{i j}$ is the decay yield from nuclide $i$ to nuclide $j$ and $f_{i j}$ is the reaction yield, $\bar{\phi}$ is the collapsed one-group flux, and $\sigma_{i}$ is the one-group flux-weighted reaction cross-section. Thus, individual transition coefficients can be thought of as relative indicators of the rates of production and loss into individual nuclides. Evaluated this way, it is evident in Tables 7 and 8 (which list the most significant gain and loss transition coefficients for ${ }^{154} \mathrm{Eu}$ and ${ }^{155} \mathrm{Eu}$ ) that neutron capture is by far the dominant gain and loss channel for both species.

Table 7: Reaction-based gain and loss channels for ${ }^{154} \mathrm{Eu}$, based on the ORIGEN transition matrix for a CANDU 28-element assembly at the start of irradiation $\left(0 \frac{\mathrm{MWd}}{\mathrm{MTU}}\right)$. (Note that only the 5 most significant transitions are given.)

\begin{tabular}{cc|cc}
\hline \multicolumn{2}{c|}{${ }^{154} \mathrm{Eu}$ gains } & \multicolumn{2}{c}{${ }^{154} \mathrm{Eu}$ losses } \\
Reaction & $a_{i j}$ & Reaction & $a_{i j}$ \\
\hline${ }^{153} \mathrm{Eu}(n, \gamma){ }^{154} \mathrm{Eu}$ & $1.1038 \mathrm{E}+02$ & ${ }^{154} \mathrm{Eu}(n, \gamma){ }^{155} \mathrm{Eu}$ & $6.6322 \mathrm{E}+02$ \\
${ }^{249} \mathrm{Cf}(n, f){ }^{154} \mathrm{Eu}$ & $3.5425 \mathrm{E}-03$ & ${ }^{154} \mathrm{Eu}(n, n){ }^{154 \mathrm{~m}} \mathrm{Eu}$ & $5.5964 \mathrm{E}-02$ \\
${ }^{243} \mathrm{Cm}(n, f){ }^{154} \mathrm{Eu}$ & $7.0951 \mathrm{E}-04$ & ${ }^{154} \mathrm{Eu}(n, 2 n){ }^{153} \mathrm{Eu}$ & $2.1322 \mathrm{E}-03$ \\
${ }^{242} \mathrm{Am}(n, f){ }^{154} \mathrm{Eu}$ & $6.3834 \mathrm{E}-04$ & ${ }^{154} \mathrm{Eu}(n, p){ }^{154} \mathrm{Sm}$ & $6.1756 \mathrm{E}-06$ \\
${ }^{251} \mathrm{Cf}(n, f){ }^{154} \mathrm{Eu}$ & $3.6207 \mathrm{E}-04$ & ${ }^{154} \mathrm{Eu}(n, \alpha){ }^{150} \mathrm{Pm}$ & $4.3990 \mathrm{E}-08$ \\
\hline
\end{tabular}

Particularly useful in understanding the miscalculations of Eu species is a comparison of the thermalregion capture cross-sections for ${ }^{153} \mathrm{Eu}$ and ${ }^{154} \mathrm{Eu}$, shown in Figure 5. Here, the ${ }^{153} \mathrm{Eu}(n, \gamma){ }^{154} \mathrm{Eu}$ crosssection is consistent between ENDF/B-VI.8 and ENDF/B-VII.0 [14, 15]. However the ENDF/B-VII.1 evaluation reports a discrepancy of approximately $11 \%$ found with experimental values [16]; thus the $2200 \mathrm{~m} / \mathrm{s}$ thermal cross-section was corrected from 312 b to 358 bn in the newest evaluation, consistent with what is seen in Figure 5(b) However, given the consistency between ENDF-VI.8 and ENDF-VII.0, this can be ruled out as the source of the discrepancy in ${ }^{154} \mathrm{Eu}$ inventories.

Likely at cause for the over-prediction is the ${ }^{154} \mathrm{Eu}(n, \gamma){ }^{155} \mathrm{Eu}$ capture cross-section, which is approximately $40 \%$ lower for the $2200 \mathrm{~m} / \mathrm{s}$ evaluation for ENDF-VII.0 compared to ENDF-VI.8. The $0.0254 \mathrm{eV}$ cross-section in ENDF/B-VI.8 is based on evaluations by Sekine et. al., indicating a value of 1840 barns 


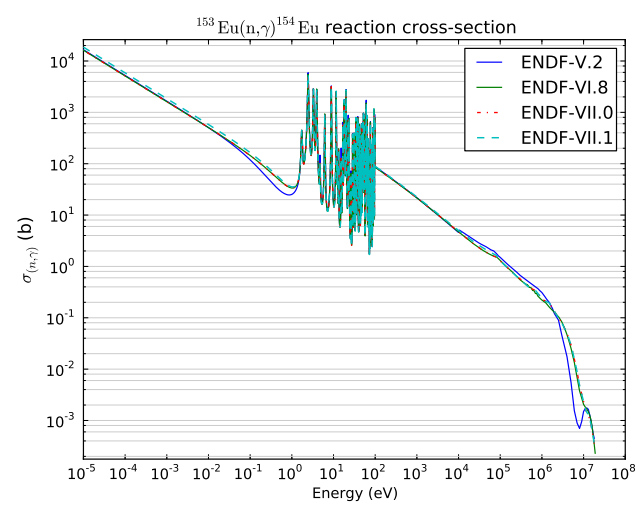

(a) ${ }^{153} \mathrm{Eu}(\mathrm{n}, \gamma){ }^{154} \mathrm{Eu}$ cross-section

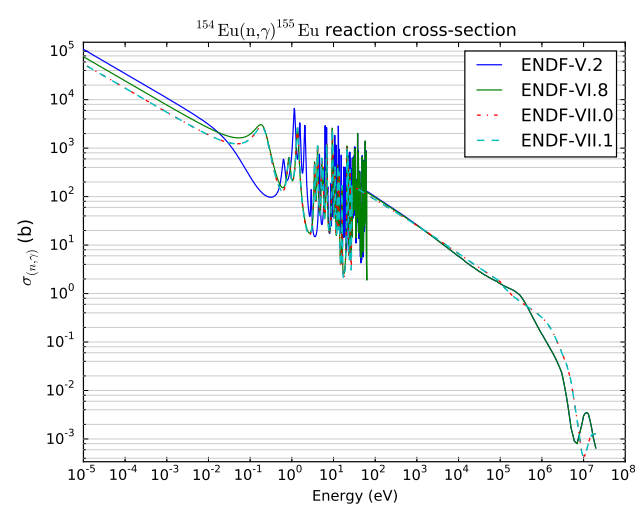

(c) ${ }^{154} \mathrm{Eu}(\mathrm{n}, \gamma){ }^{155} \mathrm{Eu}$ cross-section

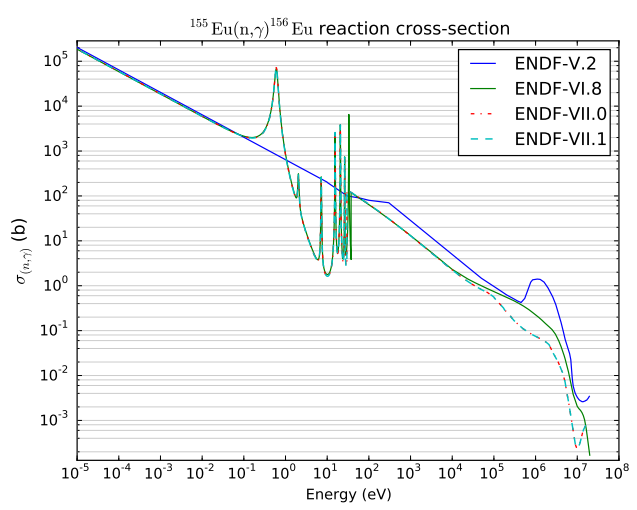

(e) ${ }^{155} \mathrm{Eu}(\mathrm{n}, \gamma){ }^{156} \mathrm{Eu}$ cross-section

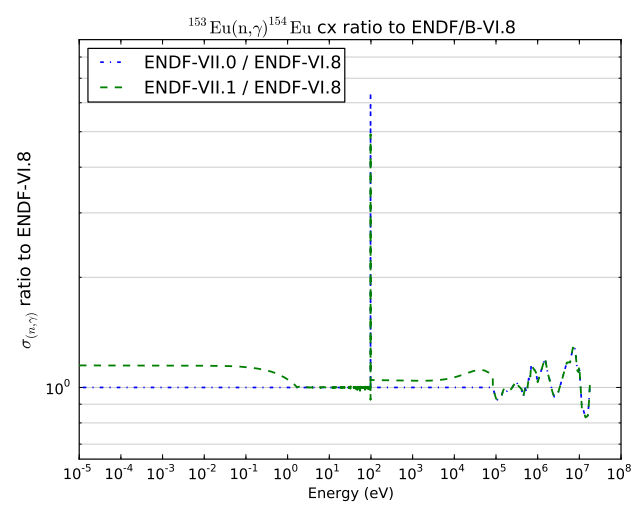

(b) ${ }^{153} \mathrm{Eu}(\mathrm{n}, \gamma){ }^{154} \mathrm{Eu}$ cross-section ratios

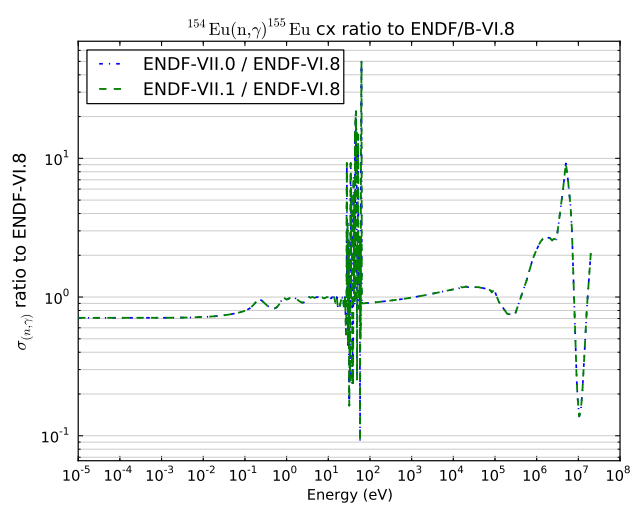

(d) ${ }^{154} \mathrm{Eu}(\mathrm{n}, \gamma){ }^{155} \mathrm{Eu}$ cross-section ratios

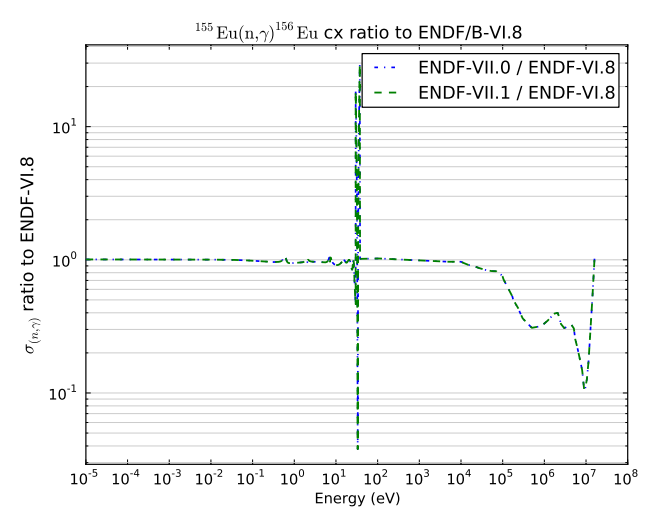

(f) ${ }^{155} \mathrm{Eu}(\mathrm{n}, \gamma){ }^{156} \mathrm{Eu}$ cross-section ratios

Figure 5: Radiative capture $(\mathrm{n}, \gamma)$ cross-sections for several ENDF library evaluations (left) and capture cross-section ratios to ENDFVI.8 data (right) for ${ }^{153} \mathrm{Eu},{ }^{154} \mathrm{Eu}$, and ${ }^{155} \mathrm{Eu}$. [14 15] 
Table 8: Reaction-based gain and loss channels for ${ }^{155} \mathrm{Eu}$, based on the ORIGEN transition matrix for a CANDU 28-element assembly at the start of irradiation $\left(0 \frac{\mathrm{MWd}}{\mathrm{MTU}}\right)$. (Note that only the 5 most significant transitions are given.)

\begin{tabular}{cc|cc}
\hline \multicolumn{2}{c}{${ }^{155} \mathrm{Eu}$ gains } & \multicolumn{2}{c}{${ }^{155} \mathrm{Eu}$ losses } \\
Reaction & $a_{i j}$ & Reaction & $a_{i j}$ \\
\hline$\left.{ }^{154} \mathrm{Eu}(n, \gamma)\right)^{155} \mathrm{Eu}$ & $6.6322 \mathrm{E}+02$ & ${ }^{155} \mathrm{Eu}(n, \gamma){ }^{156} \mathrm{Eu}$ & $1.4438 \mathrm{E}+03$ \\
$\left.{ }^{249} \mathrm{Cf}(n, f)\right)^{155} \mathrm{Eu}$ & $4.2343 \mathrm{E}-02$ & $\left.{ }^{155} \mathrm{Eu}(n, 2 n)\right)^{154} \mathrm{Eu}$ & $3.0927 \mathrm{E}-04$ \\
${ }^{242} \mathrm{Am}(n, f){ }^{155} \mathrm{Eu}$ & $1.0235 \mathrm{E}-02$ & ${ }^{155} \mathrm{Eu}(n, 2 n){ }^{154 m} \mathrm{Eu}$ & $1.2106 \mathrm{E}-04$ \\
${ }^{243} \mathrm{Cm}(n, f){ }^{155} \mathrm{Eu}$ & $9.5522 \mathrm{E}-03$ & ${ }^{155} \mathrm{Eu}(n, p){ }^{155} \mathrm{Sm}$ & $3.5362 \mathrm{E}-06$ \\
${ }^{251} \mathrm{Cf}(n, f){ }^{155} \mathrm{Eu}$ & $7.1689 \mathrm{E}-03$ & ${ }^{155} \mathrm{Eu}(n, \alpha){ }^{152 \mathrm{~m}} \mathrm{Pm}$ & $1.6364 \mathrm{E}-06$ \\
\hline
\end{tabular}

for the radiative capture cross-section [17]. The evaluation used in ENDF/B-VII.0 cites Mughabghab for an evaluated thermal capture cross-section of 1348.5 barns [18], however no mention is made of the prior evaluation by Sekine et. al. [17]. Further, File $2(\mathrm{MF}=2)$ of the ENDF/B-VII.0 library gives a calculated thermal capture cross-section of 1841.6 barns [14], which is $36.6 \%$ higher than the evaluated value and consistent with Sekine [17]. Thus, given this and the better observed agreement seen for ${ }^{154} \mathrm{Eu}$ when using the ENDF/B-VI.8 library, it appears likely that the ENDF/B-VII.0 and VII.1 libraries are in error for the ${ }^{154} \mathrm{Eu}$ thermal capture cross-section, which would thus explain the observed discrepancy.

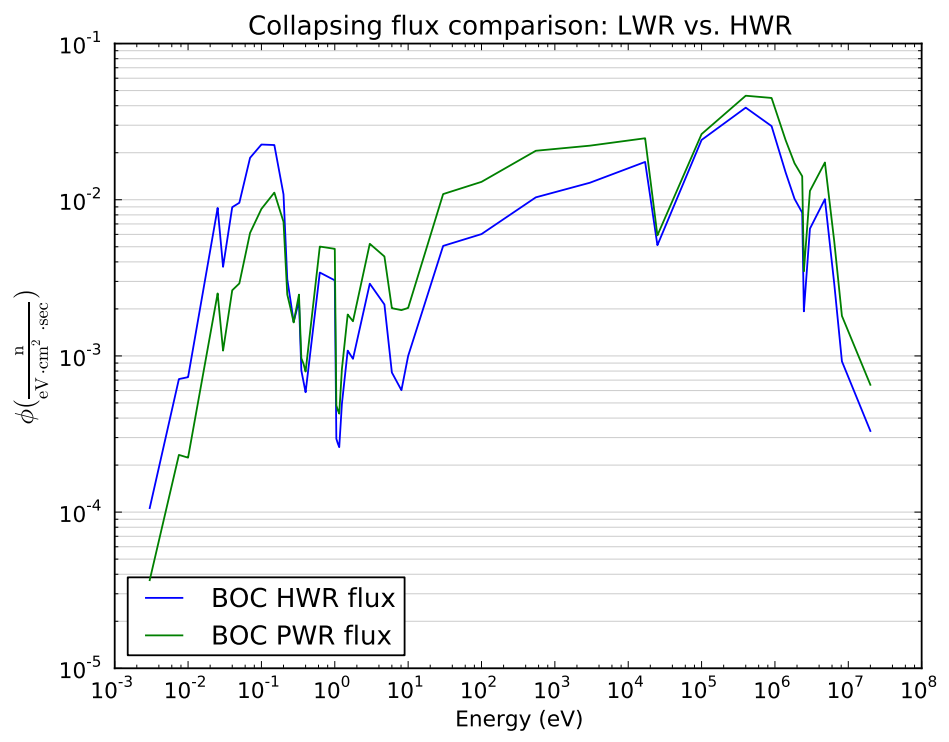

Figure 6: Distribution of the 49-group collapsing flux used at the beginning-of-core for a typical PWR assembly (Westinghouse 17x17) versus a CANDU PHWR (28-element) assembly. Note the shift of the neutron population away from the resonance region and toward the thermal region for heavy-water spectra compared to a corresponding light-water reactor spectrum.

Further compounding this issue is the fact that the highly-thermalized neutron energy spectrum for heavy-water moderators heightens the sensitivity to these cross-section differences compared with lightwater spectra, as seen by the representative beginning-of-core assembly-average flux profile shown in Fig- 
ure 6 Here, the average thermal-region neutron population for a HWR is nearly double that of a typical LWR (calculated for a Westinghouse 17x17 PWR assembly). Thus, the heavy-water lattice models are arguably more sensitive to the observed differences in the thermal-region absorption cross-sections (while being subsequently less sensitive to changes in the resonance region).

Meanwhile, ${ }^{155} \mathrm{Eu}$ is not accurately predicted by any of the libraries. Here, the production is too high for ENDF-B/V and too low for ENDF/B-VII.0. Looking at the production of ${ }^{155} \mathrm{Eu}$ via the neutron absorption from the ${ }^{154} \mathrm{Eu}(n, \gamma){ }^{155} \mathrm{Eu}$ reaction does not appear to immediately explain the discrepancy. ENDF/B-V.2 contains no resonance information; the cross-section is interpolated through the resonance region, likely explaining the over-prediction for ENDF-V libraries. Conversely, the ENDF/B-VII.0 evaluation reports a thermal capture cross-section of 3,758.4 barns, with a resonance integral of 15,527.5 barns [14]; identical values are reported for ENDF/B-VII.1 [16]. The ENDF/B-VI.8 evaluation reports a thermal capture crosssection of 3,760.22 barns with a resonance integral of 15,288 barns [15].

Given the similarities between the ENDF-VI.8 and VII.0 evaluations, it appears unlikely that the ${ }^{155} \mathrm{Eu}(\mathrm{n}, \gamma)^{156} \mathrm{Eu}$ cross-section is at issue, but rather that there may be other production channels at issue (i.e., neutron capture by ${ }^{154} \mathrm{Sm}$, the dominant loss channel with a transition coefficient of $3.3521 \mathrm{~s}^{-1}$, drives $\beta^{-}$decay by ${ }^{155} \mathrm{Sm}$ into ${ }^{155} \mathrm{Eu}$ ). This hypothesis is further supported by the differences in the evaluation of the ${ }^{154} \mathrm{Sm}(n, \gamma){ }^{155} \mathrm{Sm}$ reaction between ENDF/B-VI.2 and ENDF/B-VII.0 and VII.1, wherein the thermal capture cross-section for the latter evaluations are approximately $10 \%$ higher than the ENDF/B-VI.2 evaluation, thus driving a larger decay source term by ${ }^{155} \mathrm{Sm}$ into ${ }^{155} \mathrm{Eu}$.

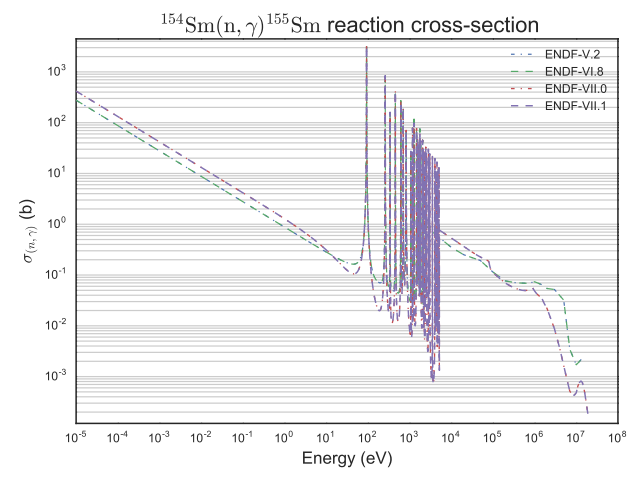

(a) ${ }^{154} \mathrm{Sm}(\mathrm{n}, \gamma){ }^{155} \mathrm{Sm}$ cross-section

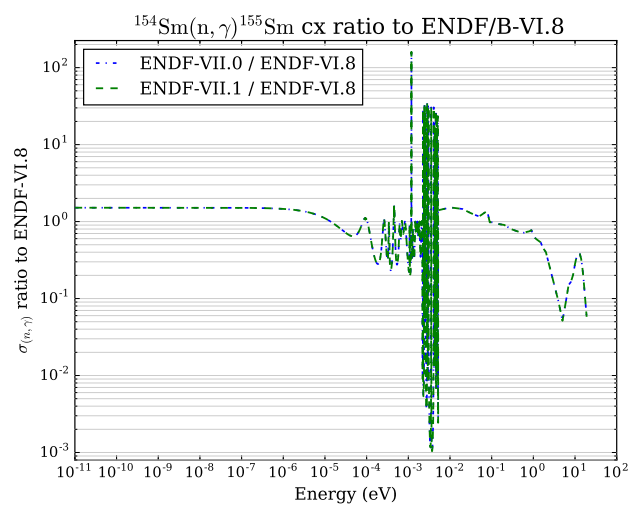

(b) ${ }^{154} \mathrm{Sm}(\mathrm{n}, \gamma){ }^{155} \mathrm{Sm}$ cross-section ratios

Figure 7: Radiative capture $(\mathrm{n}, \gamma)$ cross-sections for several ENDF library evaluations (left) and capture cross-section ratios to ENDFVI.8 data (right) for ${ }^{154} \mathrm{Sm}$. [14] 15]

Ultimately, while the issue of possible sources of the observed ${ }^{155} \mathrm{Eu}$ discrepancy resulting from changes to nuclear data evaluations merits further study, the issue was determined to be beyond the scope of this study.

\section{ORIGEN library performance comparison}

With the general validation of the new lattice used for lattice physics transport, the performance of the new ORIGEN libraries generated from these calculations can now be benchmarked in comparison to the 
previous version of the CANDU libraries distributed with SCALE (based on WIMS transport calculations). Since the older, WIMS-AECL based ORIGEN libraries were available, they were used to directly calculate the isotopic inventories.

\subsection{8-element library benchmarks}

Two different 28-element ORIGEN libraries are benchmarked. The first (referred to as the "unmodified" library) was created using the default 238-group AMPX master multi-group library using TRITON/NEWT with ENDF/VII.0 cross-sections. The second library (referred to as the "modified" library) uses a modified AMPX master multi-group library for ${ }^{154} \mathrm{Eu}$, employing modified cross-sections for the thermal-region neutron capture reaction (discussed previously in Section 5.3). Two benchmarks are conducted using both libraries, corresponding to the two power histories provided: an inferred outer element power history, and the nominal (assembly-average) power history.

The outer element power history benchmark aims to test the accuracy of the new ORIGEN library by evaluating the library using the same power history as the fuel element used for the destructive analysis benchmark. This benchmark is not directly appropriate because the assembly is using a power history from a particular zone applied across an assembly-averaged spectrum. As a result, nuclides that are strongly influenced by local variations in the flux spectrum (i.e., nuclides whose primary or only production channel is through neutron capture reactions) will suffer in terms of accuracy, while less impact will be seen in isotopes where the dominant production channel is through fission yield (be it either direct yield or accumulated yield through decay).

In order to provide a sensitivity assessment of the library for this benchmark, a modified version of the outer element power history (Table 1 w was also employed. This modification consisted of increasing the estimated outer element power (here, used as assembly-averaged power in the ORIGEN depletion benchmark) by $3 \%$ to provide a better match for the pin burnup (estimated primarily through the depletion of ${ }^{235} \mathrm{U}$ ). As with the outer element power benchmark, the modified outer power benchmark is not directly appropriate because a power history from a specific zone is applied across an assembly-averaged spectrum. However, here the burnup is modified in an attempt to produce a more accurate inventories to compensate for the assembly-averaged spectral shape.

\subsubsection{Outer element power history benchmark}

This benchmark was conducted using both modified and unmodified ORIGEN libraries produced by the new lattice design and compared to the old CANDU 28-element library. The new CANDU 28-element library was generated by irradiating the 28-element lattice at a constant specific power of $25 \mathrm{MW} / \mathrm{MTU}$ applied over 461 days to reach an exit burnup of 11,520 $\mathrm{MWd} / \mathrm{MTU}$, the same as the previous library. The previous library consisted of 8 library positions, however, the new library used 21 library positions. The results of this benchmark are shown in Figures 8 and 9

The new libraries produced similar major actinide inventories as the old library, with most of the results residing inside the experimental uncertainty. It should be noted that neither the old or new ORIGEN library produced an ${ }^{235} \mathrm{U}$ value that resided within the experimental uncertainty. Compared to the experimental value the new modified library produced better correspondence for ${ }^{236} \mathrm{U}$ and ${ }^{241} \mathrm{Pu}$. There was a large decrease in accuracy for ${ }^{244} \mathrm{Cm}$, compared to the nominal case, indicating the incorrect flux shape and distribution. Key burnup indicators ${ }^{134} \mathrm{Cs}$ and ${ }^{137} \mathrm{Cs}$ and key fission products ${ }^{237} \mathrm{~Np}$ and ${ }^{241} \mathrm{Am}$ were within the experimental uncertainty bounds for both modified and unmodified libraries. 


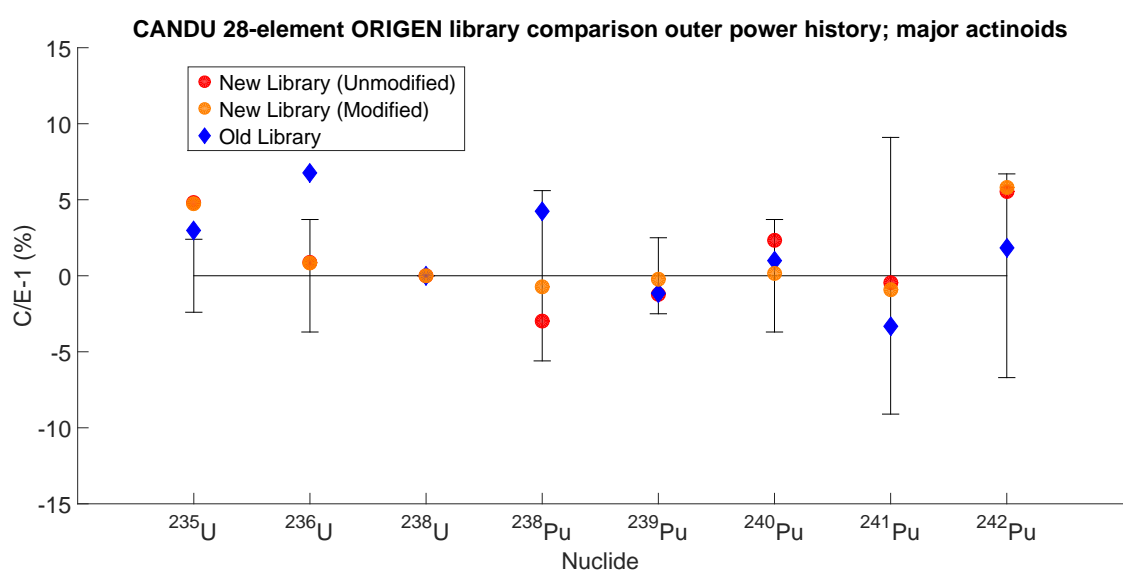

Figure 8: CANDU 28-element ORIGEN outer element power history comparison; major actinoids

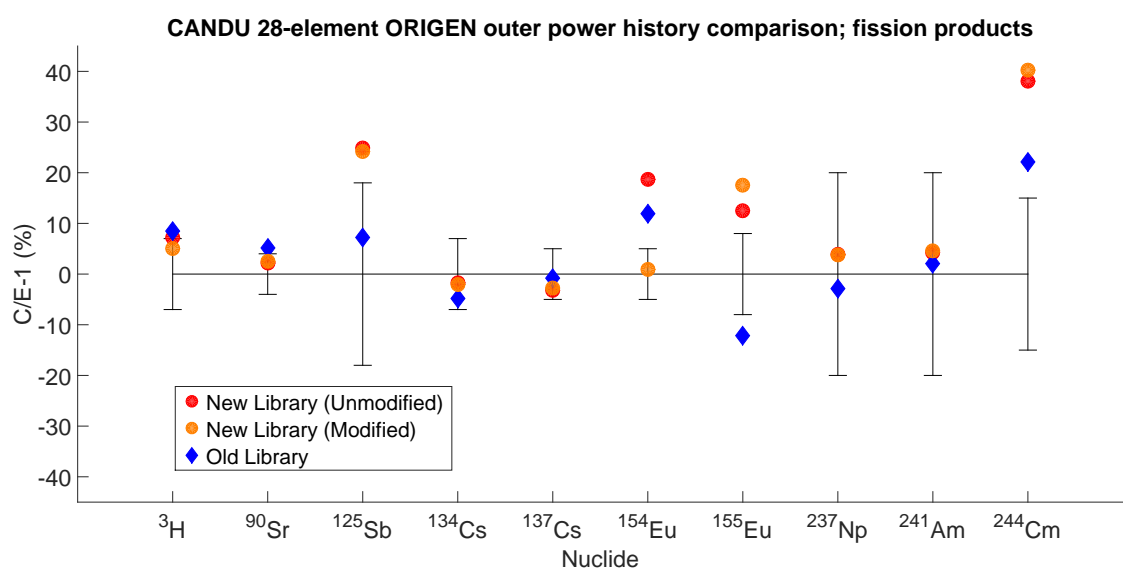

Figure 9: CANDU 28-element ORIGEN outer element power history comparison; minor actinoids and fission products

\subsubsection{Modified outer element power history benchmark}

This benchmark used both the AMPX modified and unmodified libraries with a modified outer element power history. This power history (the "outer" power history in Table 1 adjusted upward by 3\%) was adjusted to ensure accurate inventory of ${ }^{235} \mathrm{U}$. This benchmark compares the assembly-averaged values from ORIGEN to the outer element assay. The goal of the benchmark was to approximate the correct burnup such that the calculated assembly average ${ }^{235} \mathrm{U}$ value matched the outer pin assay. The results of the benchmark are provided in Figures 10 and 11 .

The modified AMPX library shows better correspondence than the WIMS-based library for many of the major actinoids. The modified library shows significant improvement in the calculation of ${ }^{238} \mathrm{Pu}$ over the WIMS-based library, which was calculated outside the experimental uncertainty. ${ }^{242} \mathrm{Pu}$ was not accurately predicted for any library, indicating that the spectrum is not correct.

Notably, the new modified AMPX library does exhibit significantly better correspondence for ${ }^{154} \mathrm{Eu}$ as a result of the modified thermal capture cross-section. Several key burnup indicators, such as ${ }^{134} \mathrm{Cs},{ }^{137} \mathrm{Cs}$, 


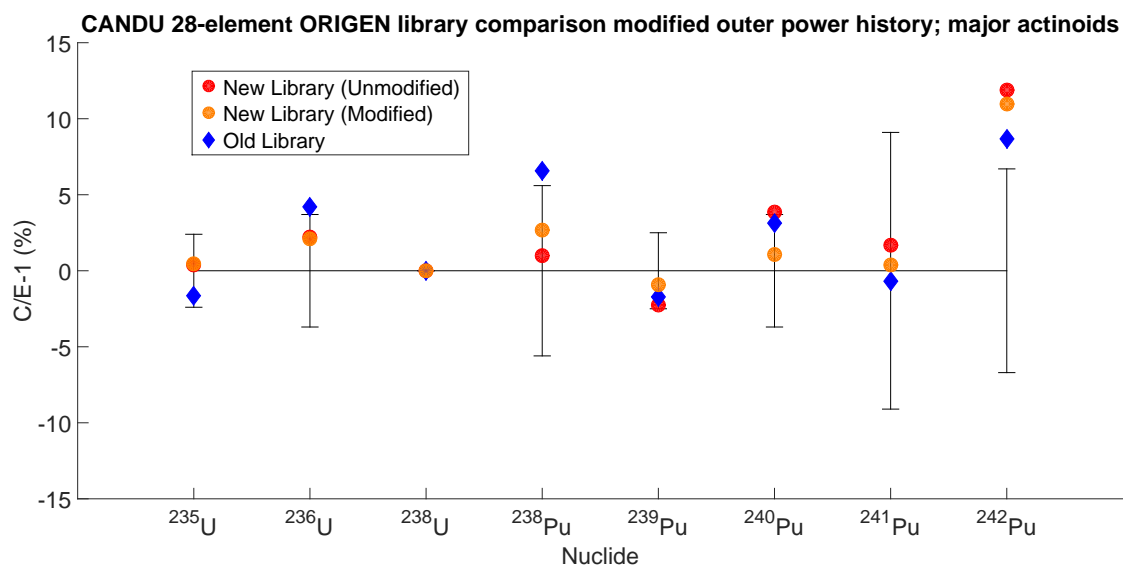

Figure 10: CANDU 28-element ORIGEN library comparison modified outer element power history; major actinoids. Note that benchmark inventories are rescaled from edge element values (per [5]) to produce an assembly-averaged value.

and ${ }^{237} \mathrm{~Np}$ are calculated inside the experimental uncertainty. However, ${ }^{244} \mathrm{Cm}$ has a large degree of error, further indicating that while the correct burnup is achieved, the flux shape is incorrect (again due to applying an assembly-averaged flux shape to a specific fuel zone).

\subsection{7-element ORIGEN library benchmark}

Figure 12 gives a benchmark comparison for major actinide species calculated in ORIGEN using the prior WIMS-based library distributed with SCALE and the newly-developed ENDF/B-VII.0-based library. All values for both libraries lie within the experimental uncertainty. However, the new library produced results that were closer to the experimental value for all of the major actinoids with the exception of ${ }^{241} \mathrm{Pu}$. Here, assembly-averaged cross-sections are used by ORIGEN to produce assembly-averaged isotopic inventories, which can then be directly compared to the 37-element radiochemical assay benchmark.

\section{Conclusion}

Overall, data from the available benchmarks thus suggest that the new CANDU lattices should provide satisfactory accuracy in line with the levels of accuracy of other provided SCALE fuel lattice templates. While all of the evaluated major actinoids for each CANDU fuel assembly lattice models presented here fit within the experimental uncertainty, some models performed better than others.

\subsection{Effect of cross-section libraries on isotopic benchmarks as evaluated in lattice physics transport models}

For the 37-element bundle, ENDF/B-VII.0 libraries outperform the ENDF/V libraries with respect to predictions of plutonium isotopes. For the 28 -element bundle, all libraries produced similar results for the uranium isotopes. ENDF/B-VII.0 and ENDF/B-VI produced better results for all the plutonium isotopes except for ${ }^{242} \mathrm{Pu}$. ENDF/B-VI produced the best results for ${ }^{238} \mathrm{Pu}$ and ${ }^{240} \mathrm{Pu}$. All libraries produced results inside the experimental uncertainty for the major actinoids. Additionally, the ENDF/B-VII.0 library performed comparably or better than the prior WIMS-AECL model with significant improvement in correspondence for ${ }^{238} \mathrm{Pu}$ and ${ }^{242} \mathrm{Pu}$ inventories. 


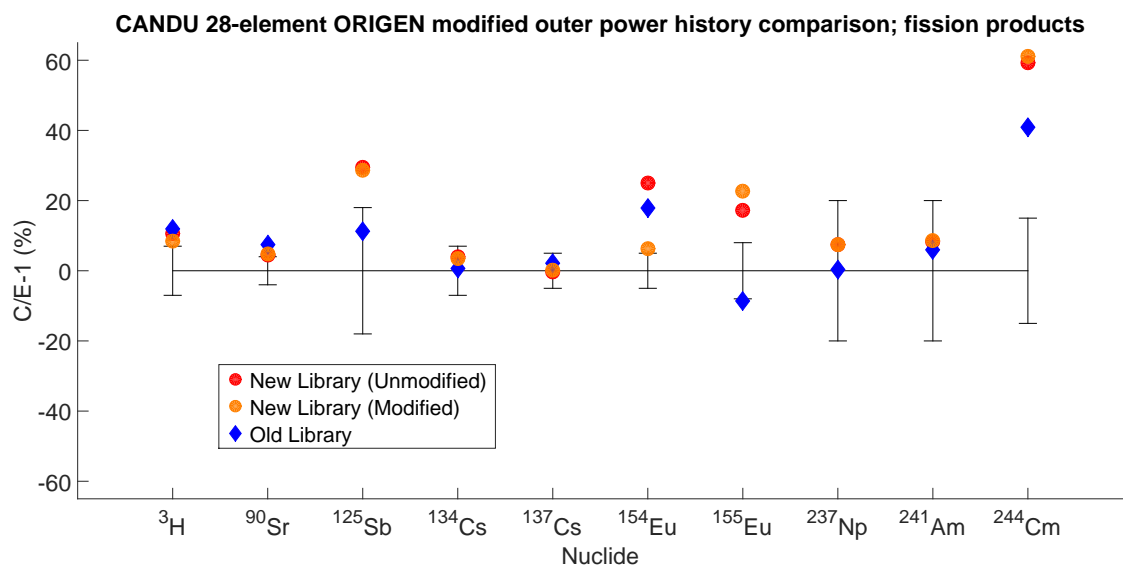

Figure 11: CANDU 28-element ORIGEN library comparison modified outer element power history; fission products and minor actinoids

For fission product comparisons, the ENDF/B-V and ENDF/B-VII.0-based libraries generally performed similarly, with ENDF/B-VII.0 showing slightly worse correspondence to experimental assay data for ${ }^{154} \mathrm{Eu}$ but slightly better for ${ }^{155} \mathrm{Eu}$. For both of these isotopes, the calculated values are outside of the experimental uncertainties for all cross-section libraries evaluated and show substantially poorer agreement compared with the WIMS-AECL model. This is likely due to the cross-section disagreements outlined previously. Prior studies on the effect of cross-section data and cross-section processing options (e.g., NITAWL versus CENTRM) found effects on the order of $2-3 \%$ for ${ }^{154} \mathrm{Eu}$ in LWR lattices. In this study however, the use ENDF/B-VII.0 (from ENDF/B-VI.2) showed up to a $20 \%$ difference in predicted inventories for ${ }^{155} \mathrm{Eu}$, one of the more significantly changed nuclides between the cross-section libraries [12]. ENDF/B-VI also had similar performance to ENDF/VII.0 for many of the fission products. While the ENDF/B-VI library did produce accurate results for ${ }^{154} \mathrm{Eu}$, it performed far worse for ${ }^{155} \mathrm{Eu}$, over-predicting this nuclide by $15 \%$, a significantly poorer agreement than both ENDF/B-VII.0 and ENDF/B-V.2-based calculations.

For minor actinoids, while ${ }^{237} \mathrm{~Np}$ showed increased correspondence from ENDF/B-V to ENDF/B-VII.0, other minor actinoids such as ${ }^{241} \mathrm{Am}$ and ${ }^{244} \mathrm{Cm}$ showed slightly poorer correspondence. For all of the minor actinoids, the move to the ENDF/B-VII.0 libraries appears to create a bias in favor of higher actinide production (i.e., more of the transuranic species are produced for ENDF/B-VII.0 libraries), likely due to the improved resonance-region resolution for these libraries [12]. This trend is likewise supported in the behavior of the plutonium isotopes, where a slight decline in ${ }^{239} \mathrm{Pu}$ is observed in favor of the higher plutonium isotopes in inventories generated using ENDF/B-VII.0. ENDF/B-VI produced poorer results for minor actinoids ${ }^{241} \mathrm{Am}$ and ${ }^{237} \mathrm{~Np}$, however, it did see an improvement in ${ }^{244} \mathrm{Cm}$ over ENDF/B-VII.0.

\subsection{Performance of the new SCALE-based lattices compared to the prior WIMS-AECL library}

In general, the new ENDF/B-VII.0-based SCALE lattices showed improvements in major actinide predictions for both lattices compared to the prior WIMS-AECL model, as well as in predictions of ${ }^{237} \mathrm{~Np}$. In general, ${ }^{154} \mathrm{Eu}$ and ${ }^{237} \mathrm{~Np}$ showed the most marked differences in the two models, likely due to improvements in cross-section treatments inherent to the ENDF/B-VII.0 cross-section libraries [12]. For most measured isotopes, the calculated inventories were well within experimental uncertainties. Predictions for fission products fared slightly worse in the new lattice models, particularly for ${ }^{155} \mathrm{Eu}$, which is likely due to the 


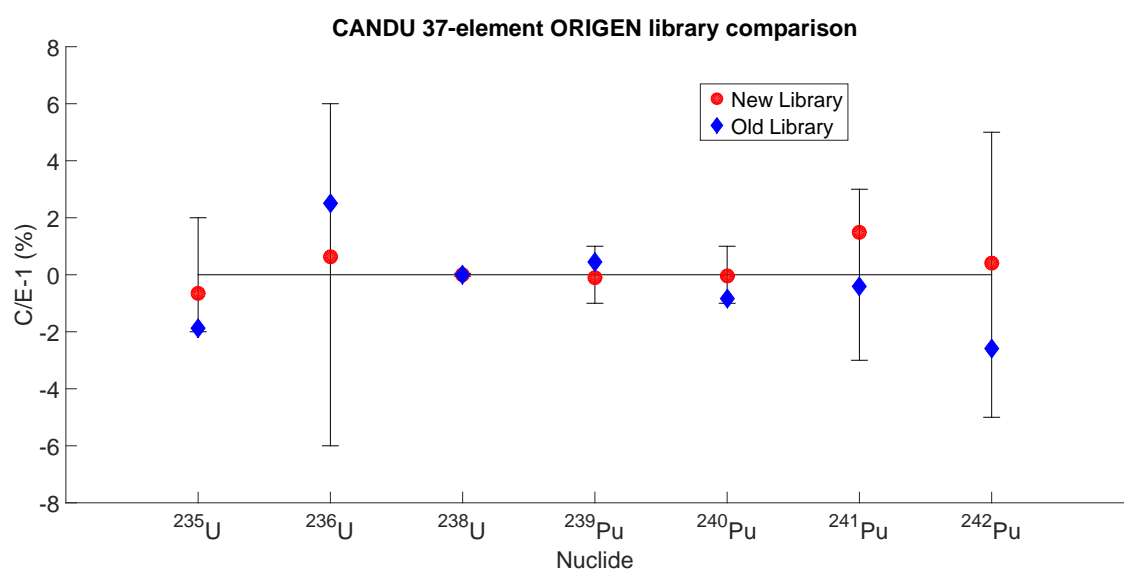

Figure 12: CANDU 37-element ORIGEN library comparison

adjustment of the ${ }^{154} \mathrm{Eu}$ cross-section. However, for most other fission product and minor actinoid isotopes, these differences were small and generally still within experimental uncertainties.

\subsubsection{CANDU 28-element lattice}

It is likely that the WIMS-AECL inventories were derived by normalizing the outer element power to a specific pin. However, the power history for a particular element (i.e., an individual fuel pin) is not usually available. In practice, the nominal power case (Section 5.1.1) will be used.

For the major actinoids, the new lattice using the nominal power history performed similarly to the WIMS-AECL model. While both models generally captured uranium and plutonium isotopes within experimental uncertainty, the new 28-element lattice model generally showed slightly improved agreement with experimental values compared to the prior WIMS results for these species, including for ${ }^{238} \mathrm{Pu}$, which was consistently under-predicted for both the WIMS lattice and the new SCALE-based lattices employing modern nuclear data evaluations.

The accuracy of the minor actinoids and fission products of the new SCALE library were similar to the WIMS-AECL model, with the exception of ${ }^{154} \mathrm{Eu}$. The specific use of ENDF/B-VI.8 cross-sections for ${ }^{154} \mathrm{Eu}$ saw a significant increase in the reported accuracy of the ORIGEN library (by about 10\%). Additionally, ${ }^{237} \mathrm{~Np}$ saw a increase in accuracy of $10 \%$ in the new ENDF/B-VII.0-based SCALE model. ${ }^{155} \mathrm{Eu}$ was not accurately reflected by any library iteration; the inclusion of the more accurate ${ }^{154} \mathrm{Eu}$ capture cross-section lead to substantial over-predictions in the ${ }^{155} \mathrm{Eu}$ inventory (outside experimental uncertainty bounds), while the WIMS-AECL-based ORIGEN library significantly under-predicted ${ }^{155} \mathrm{Eu}$ inventories (again, outside of experimental uncertainty). It is hypothesized that this discrepancy may reflect the influence of other changes to nuclear data evaluations, such as the thermal neutron capture cross-section of ${ }^{154} \mathrm{Sm}$, warranting further investigation beyond the scope of this study.

\subsubsection{CANDU 37-element lattice}

The new ENDF/B-VII.0-based lattice showed at least a slight improvement in all calculated inventories compared to the WIMS-AECL model except for ${ }^{241} \mathrm{Pu}$. A notable improvement was observed for ${ }^{242} \mathrm{Pu}$, which was outside the uncertainty for the prior WIMS-AECL-based library. All calculated ENDF/B-VII.0 results lie within the experimental uncertainty. 


\section{Acknowledgments}

This work was supported by an Nuclear Energy University Programs (NEUP) grant sponsored by the U.S. Department of Energy, Office of Nuclear Energy, award number DE-NE0000737.

The authors wish to thank Ian Gauld of Oak Ridge National Laboratory, who in addition to furnishing them with the original CANDU validation studies, provided much-needed assistance and insights in determining the source of validation issues with the lattices as well as thoughtful comments on drafts of this paper. Finally, the authors would like to acknowledge Brian Ade of Oak Ridge National Laboratory, who likewise provided guidance and troubleshooting assistance in the lattice validation studies.

[1] Oak Ridge National Laboratory, SCALE: A Comprehensive Modeling and Simulation Suite for Nuclear Safety Analysis and Design, Version 6.1 (June 2011).

[2] S. Bowman, SCALE 6: Comprehensive Nuclear Safety Analysis Code System, Nuclear Technology 174 (2) (2011) 126-148.

[3] I. C. Gauld, G. Radulescu, G. Ilas, B. D. Murphy, M. L. Williams, D. Wiarda, Isotopic Depletion and Decay Methods and Analysis Capabilities in SCALE, Nuclear Technology 174 (2) (2011) 169-195.

[4] J. Tait, I. Gauld, A. H. Kerr, Validation of the ORIGEN-S Code for Predicting Radionuclide Inventories in Used CANDU Fuel, Journal of Nuclear Materials 223 (2) (1995) 109-121. URL http://www.sciencedirect.com/science/article/pii/0022311594006830\#

[5] I. Gauld, P. Carlson, K. Litwin, Production and Validation of ORIGEN-S Cross-Section Libraries for CANDU Reactor Fuel Studies, Tech. Rep. RC-1442; COG-I-95-200, Whiteshell Laboratories (October 1995).

[6] I. C. Gauld, K. A. Litwin, Verification and Validation of the ORIGEN-S Code and Nuclear Data Libraries, Tech. Rep. RC-1429 / COG-I-95-150, Atomic Energy of Canada Limited (AECL) (August 1995).

URL http://web.ornl.gov/sci/scale/pubs/rc1429.pdf

[7] M. D. DeHart, S. M. Bowman, Reactor Physics Methods and Analysis Capabilities in SCALE, Nuclear Technology 174 (2) (2011) 196-213.

[8] S. Goluoglu, L. M. Petrie, M. E. Dunn, D. F. Hollenbach, B. T. Rearden, Monte Carlo Criticality Methods and Analysis Capabilities in SCALE, Nuclear Technology 174 (2) (2011) 214-235.

[9] C. H. Chung, K. B. Oh, C. H. Kim, Y. H. Chang, S. Y. Kim, J. S. Kim, Nuclear Design Analysis of Wolsung-1 CANDU-PHW Nuclear Generating Station, Journal of the Korean Nuclear Society 10 (4) (1978) 203-213.

URL http://wWW.kns.org/jknsfile/v10/A04803288321.pdf?PHPSESSID= a078b088435d92a883ed144a4b241782

[10] S. Goluoglu, D. F. Hollenbach, N. F. Landers, L. M. Petrie, J. A. Bucholz, C. F. Weber, C. M. Hopper, The Material Information Processor for SCALE, Oak Ridge National Laboratory (June 2011).

[11] H. Smith, Modeling depletion simulations for a high-burnup, highly heterogeneous bwr fuel assembly with scale, in: PHYSOR - Advances in Reactor Physics, American Nuclear Society, 2012. 
[12] G. Ilas, I. C. Gauld, G. Radulescu, Validation of new depletion capabilities and ENDF/B-VII data libraries in SCALE, Annals of Nuclear Energy 46 (2012) 43-55.

URL http://dx.doi .org/10.1016/j . anucene.2012.03.012

[13] M. Basunia, R. Firestone, Z. Révay, H. Choi, T. Belgya, J. Escher, A. Hurt, M. Krtička, L. Szentmiklósi, B. Sleaford, N. Summers, Determination of the ${ }^{151} \mathrm{Eu}(\mathrm{n}, \gamma){ }^{152 m 1, g} \mathrm{Eu}$ and ${ }^{153} \mathrm{Eu}(\mathrm{n}, \gamma){ }^{154} \mathrm{Eu}$ Reaction Cross Sections at Thermal Neutron Energy, Nuclear Data Sheets 119 (2014) 88-90.

[14] M. Chadwick, P. Obložinský, M. Herman, N. Greene, R. McKnight, D. Smith, P. Young, R. MacFarlane, G. Hale, S. Frankle, A. Kahler, T. Kawano, R. Little, D. Madland, P. Moller, R. Mosteller, P. Page, P. Talou, H. Trellue, M. White, W. Wilson, R. Arcilla, C. Dunford, S. Mughabghab, B. Pritychenko, D. Rochman, A. Sonzogni, C. Lubitz, T. Trumbull, J. Weinman, D. Brown, D. Cullen, D. Heinrichs, D. McNabb, H. Derrien, M. Dunn, N. Larson, L. Leal, A. Carlson, R. Block, J. Briggs, E. Cheng, H. Huria, M. Zerkle, K. Kozier, A. Courcelle, V. Pronyaev, S. van der Marck, ENDF/BVII.0: Next Generation Evaluated Nuclear Data Library for Nuclear Science and Technology, Nuclear Data Sheets 107 (12) (2006) 2931-3060, Evaluated Nuclear Data File ENDF/B-VII.0. doi:http: $/ /$ dx.doi.org/10.1016/j.nds.2006.11.001

URL http://www.sciencedirect.com/science/article/pii/S0090375206000871

[15] Cross-Section Evaluation Working Group, ENDF/B-VI Summary Documentation, Tech. Rep. BNLNCS-17541 (ENDF-201), Data Library ENDF/B-VI, update 1998, by the U.S. National Nuclear Data Center on behalf of the Cross-Section Evaluation Working Group (1998).

[16] M. Chadwick, M. Herman, P. Obložinský, M. Dunn, Y. Danon, A. Kahler, D. Smith, B. Pritychenko, G. Arbanas, R. Arcilla, R. Brewer, D. Brown, R. Capote, A. Carlson, Y. Cho, H. Derrien, K. Guber, G. Hale, S. Hoblit, S. Holloway, T. Johnson, T. Kawano, B. Kiedrowski, H. Kim, S. Kunieda, N. Larson, L. Leal, J. Lestone, R. Little, E. McCutchan, R. MacFarlane, M. MacInnes, C. Mattoon, R. McKnight, S. Mughabghab, G. Nobre, G. Palmiotti, A. Palumbo, M. Pigni, V. Pronyaev, R. Sayer, A. Sonzogni, N. Summers, P. Talou, I. Thompson, A. Trkov, R. Vogt, S. van der Marck, A. Wallner, M. White, D. Wiarda, P. Young, ENDF/B-VII.1 Nuclear Data for Science and Technology: Cross Sections, Covariances, Fission Product Yields and Decay Data. Nuclear Data Sheets 112 (12) (2011) 2887-2996, special Issue on ENDF/B-VII.1 Library. doi:http://dx.doi.org/10.1016/j.nds . 2011.11.002 URL http://wWw.sciencedirect.com/science/article/pii/S009037521100113X

[17] T. Sekine, S.-I. Ichikawa, S. Baba, Triple Neutron Capture of ${ }^{153} \mathrm{Eu}$ in a Reactor: The Cross Sections of ${ }^{154} \mathrm{Eu}$ and ${ }^{155} \mathrm{Eu}$, International Journal of Radiation Applications and Instrumentation. Part A. Applied Radiation and Isotopes 38 (7) (1987) 513-516.

[18] S. F. Mughabghab, Atlas of Neutron Resonances, Fifth Edition: Resonance Parameters and Thermal Cross Sections. Z=1-100, Elsevier Science, 2006. 
[1] Oak Ridge National Laboratory, SCALE: A Comprehensive Modeling and Simulation Suite for Nuclear Safety Analysis and Design, Version 6.1 (June 2011).

[2] S. Bowman, SCALE 6: Comprehensive Nuclear Safety Analysis Code System, Nuclear Technology 174 (2) (2011) 126-148.

[3] I. C. Gauld, G. Radulescu, G. Ilas, B. D. Murphy, M. L. Williams, D. Wiarda, Isotopic Depletion and Decay Methods and Analysis Capabilities in SCALE, Nuclear Technology 174 (2) (2011) 169-195.

[4] J. Tait, I. Gauld, A. H. Kerr, Validation of the ORIGEN-S Code for Predicting Radionuclide Inventories in Used CANDU Fuel, Journal of Nuclear Materials 223 (2) (1995) 109-121. URL http://www. sciencedirect. com/science/article/pii/0022311594006830\#

[5] I. Gauld, P. Carlson, K. Litwin, Production and Validation of ORIGEN-S Cross-Section Libraries for CANDU Reactor Fuel Studies, Tech. Rep. RC-1442; COG-I-95-200, Whiteshell Laboratories (October 1995).

[6] I. C. Gauld, K. A. Litwin, Verification and Validation of the ORIGEN-S Code and Nuclear Data Libraries, Tech. Rep. RC-1429 / COG-I-95-150, Atomic Energy of Canada Limited (AECL) (August 1995).

URL http://web.ornl.gov/sci/scale/pubs/rc1429.pdf

[7] M. D. DeHart, S. M. Bowman, Reactor Physics Methods and Analysis Capabilities in SCALE, Nuclear Technology 174 (2) (2011) 196-213.

[8] S. Goluoglu, L. M. Petrie, M. E. Dunn, D. F. Hollenbach, B. T. Rearden, Monte Carlo Criticality Methods and Analysis Capabilities in SCALE, Nuclear Technology 174 (2) (2011) 214-235.

[9] C. H. Chung, K. B. Oh, C. H. Kim, Y. H. Chang, S. Y. Kim, J. S. Kim, Nuclear Design Analysis of Wolsung-1 CANDU-PHW Nuclear Generating Station, Journal of the Korean Nuclear Society 10 (4) (1978) 203-213.

URL http://www.kns.org/jknsfile/v10/A04803288321.pdf?PHPSESSID= a078b088435d92a883ed144a4b241782

[10] S. Goluoglu, D. F. Hollenbach, N. F. Landers, L. M. Petrie, J. A. Bucholz, C. F. Weber, C. M. Hopper, The Material Information Processor for SCALE, Oak Ridge National Laboratory (June 2011).

[11] H. Smith, Modeling depletion simulations for a high-burnup, highly heterogeneous bwr fuel assembly with scale, in: PHYSOR - Advances in Reactor Physics, American Nuclear Society, 2012.

[12] G. Ilas, I. C. Gauld, G. Radulescu, Validation of new depletion capabilities and ENDF/B-VII data libraries in SCALE, Annals of Nuclear Energy 46 (2012) 43-55.

URL http://dx. doi .org/10.1016/j . anucene.2012.03.012

[13] M. Basunia, R. Firestone, Z. Révay, H. Choi, T. Belgya, J. Escher, A. Hurt, M. Krtička, L. Szentmiklósi, B. Sleaford, N. Summers, Determination of the ${ }^{151} \mathrm{Eu}(\mathrm{n}, \gamma){ }^{152 m 1, g} \mathrm{Eu}$ and ${ }^{153} \mathrm{Eu}(\mathrm{n}, \gamma)^{154} \mathrm{Eu}$ Reaction Cross Sections at Thermal Neutron Energy, Nuclear Data Sheets 119 (2014) 88-90.

[14] M. Chadwick, P. Obložinský, M. Herman, N. Greene, R. McKnight, D. Smith, P. Young, R. MacFarlane, G. Hale, S. Frankle, A. Kahler, T. Kawano, R. Little, D. Madland, P. Moller, R. Mosteller, 
P. Page, P. Talou, H. Trellue, M. White, W. Wilson, R. Arcilla, C. Dunford, S. Mughabghab, B. Pritychenko, D. Rochman, A. Sonzogni, C. Lubitz, T. Trumbull, J. Weinman, D. Brown, D. Cullen, D. Heinrichs, D. McNabb, H. Derrien, M. Dunn, N. Larson, L. Leal, A. Carlson, R. Block, J. Briggs, E. Cheng, H. Huria, M. Zerkle, K. Kozier, A. Courcelle, V. Pronyaev, S. van der Marck, ENDF/BVII.0: Next Generation Evaluated Nuclear Data Library for Nuclear Science and Technology, Nuclear Data Sheets 107 (12) (2006) 2931-3060, Evaluated Nuclear Data File ENDF/B-VII.0. doi:http: //dx.doi.org/10.1016/j.nds.2006.11.001.

URL http://www.sciencedirect.com/science/article/pii/S0090375206000871

[15] Cross-Section Evaluation Working Group, ENDF/B-VI Summary Documentation, Tech. Rep. BNLNCS-17541 (ENDF-201), Data Library ENDF/B-VI, update 1998, by the U.S. National Nuclear Data Center on behalf of the Cross-Section Evaluation Working Group (1998).

[16] M. Chadwick, M. Herman, P. Obložinský, M. Dunn, Y. Danon, A. Kahler, D. Smith, B. Pritychenko, G. Arbanas, R. Arcilla, R. Brewer, D. Brown, R. Capote, A. Carlson, Y. Cho, H. Derrien, K. Guber, G. Hale, S. Hoblit, S. Holloway, T. Johnson, T. Kawano, B. Kiedrowski, H. Kim, S. Kunieda, N. Larson, L. Leal, J. Lestone, R. Little, E. McCutchan, R. MacFarlane, M. MacInnes, C. Mattoon, R. McKnight, S. Mughabghab, G. Nobre, G. Palmiotti, A. Palumbo, M. Pigni, V. Pronyaev, R. Sayer, A. Sonzogni, N. Summers, P. Talou, I. Thompson, A. Trkov, R. Vogt, S. van der Marck, A. Wallner, M. White, D. Wiarda, P. Young, ENDF/B-VII.1 Nuclear Data for Science and Technology: Cross Sections, Covariances, Fission Product Yields and Decay Data, Nuclear Data Sheets 112 (12) (2011) 2887-2996, special Issue on ENDF/B-VII.1 Library. doi:http://dx.doi.org/10.1016/j.nds. 2011.11 .002 .

URL http://www.sciencedirect.com/science/article/pii/S009037521100113X

[17] T. Sekine, S.-I. Ichikawa, S. Baba, Triple Neutron Capture of ${ }^{153} \mathrm{Eu}$ in a Reactor: The Cross Sections of ${ }^{154} \mathrm{Eu}$ and ${ }^{155} \mathrm{Eu}$, International Journal of Radiation Applications and Instrumentation. Part A. Applied Radiation and Isotopes 38 (7) (1987) 513-516.

[18] S. F. Mughabghab, Atlas of Neutron Resonances, Fifth Edition: Resonance Parameters and Thermal Cross Sections. Z=1-100, Elsevier Science, 2006. 